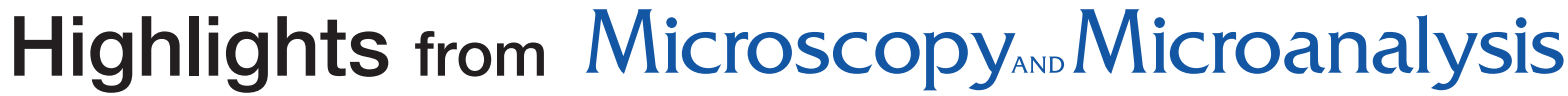

\section{Techniques and Materials Applications}

Fast Atomic-Scale Elemental Mapping of Crystalline Materials by STEM Energy-Dispersive X-Ray Spectroscopy Achieved With Thin Specimens by P Lu, R Yuan, and JM Zuo, Microsc Microanal 23(1) (2017) 145-54

Elemental mapping at atomic-scale by scanning transmission electron microscopy (STEM) using energy-dispersive x-ray spectroscopy (EDS) provides a powerful real-space approach to chemical characterization of crystal structures. However, applications of this technique have been limited by inefficient $\mathrm{X}$-ray emission and collection, which require long acquisition times. Here we describe a fast atomic-scale STEM-EDS imaging technique that decreases the acquisition time by more than 100 times by using a lattice-vector translation method. This technique provides atomic-scale elemental maps averaged over areas of $\sim$ few $10 \mathrm{~nm}^{2}$ with the acquisition time of $\sim 2 \mathrm{~s}$ or less. Phenomenological modeling shows that the localization of $\mathrm{X}$-ray counts to atomic columns is a key reason for the success of the technique. Multislice image simulations show that the X-ray localization can be achieved by choosing a specimen with a thickness less than $\sim 20 \mathrm{~nm}$ for [001] $\mathrm{SrTiO}_{3}$ when using $200 \mathrm{keV}$ electrons. The fast EDS mapping provides a means for studying electron beam sensitive materials and temporal, atomic-scale mapping of crystal structure/chemistry for investigations of dynamic phenomena in materials.
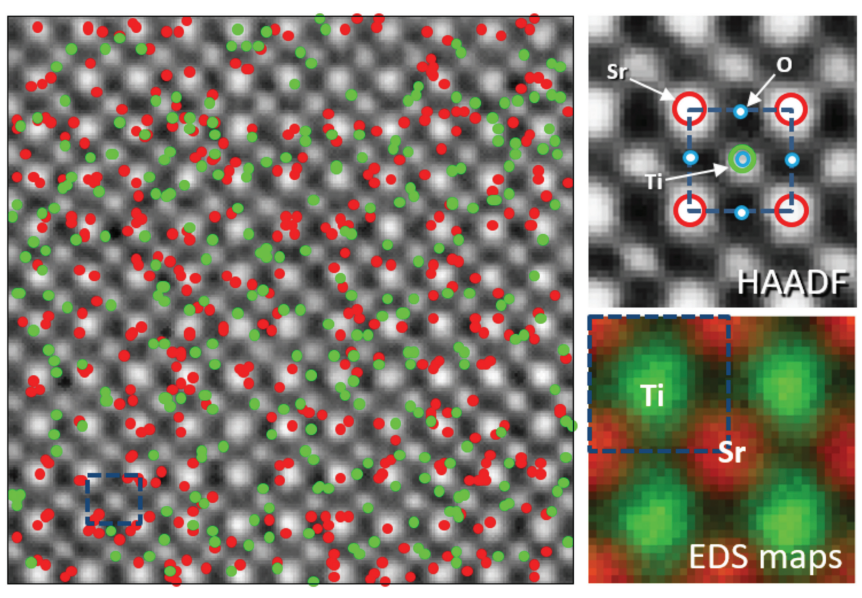

Fast atomic-scale STEM-EDS mapping demonstrated with a SrTiO3 (STO) crystal. Raw X-ray count maps of Sr L and K (red dots) and Ti K (green dots) obtained by STEM-EDS, with a total acquisition time of $\sim 2 \mathrm{~s}$, overlaid on the reference HAADF image (the left panel). The lattice-vector translation (or lattice-averaging) method is used to provide averaged EDS maps. The square in the images represents the [001] projected STO cubic unit cell with a lattice constant of $0.39 \mathrm{~nm}$.

\section{Materials Applications}

Atomic-Scale Analytical Tomography by TF Kelly, Microsc Microanal 23(1) (2017) 34-45

The concept of atomic-scale tomography (AST) was proposed in the past decade as a technique that could provide the position and identity of all atoms in a structure of large volume (up to billion atoms) with high precision. AST would use atom probe tomography (APT) coupled with (scanning) transmission electron microscopy ((S)TEM) to eliminate image aberrations and would employ a nominal 100\% detection of the atoms. There is also a rich array of analytical information that can be brought to bear on such tomographs. In this paper, some of these types of analytical information are considered and implications are explored. The advantages and disadvantages of needle-shaped specimens are described. Coupling electron diffraction information with atom probe crystallography offers synergies for structural understanding. Adding chemical information from electron energy loss spectroscopy (EELS) is especially important for augmentation of the atomicscale compositional mapping from AST and integrating with computational materials science and computed properties. This fuller realm of analytical information coupled to structural information may be called atomic-scale analytical tomography (ASAT).

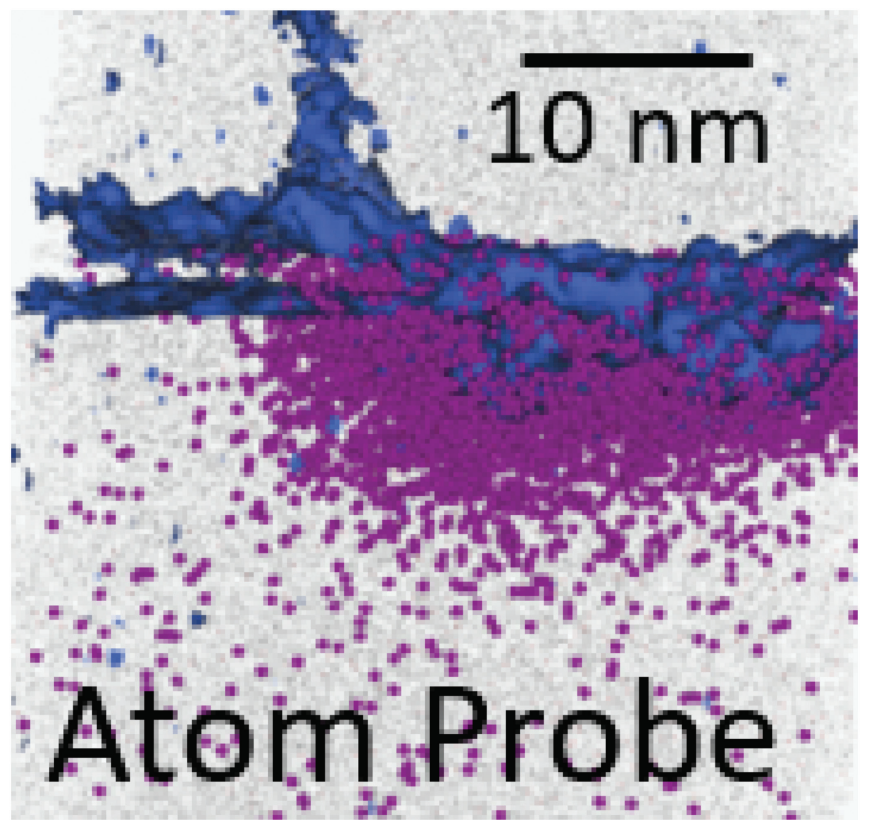

Atomic-scale analytical tomography (ASAT) uses the full realm of analytical and structural information available in a sample. 


\section{Microscopy мND $_{\text {N }}$ Microanalysis}

\section{Biological Applications}

Automatic Biological Cell Counting Using a Modified Gradient Hough Transform by E Denimal, A Marin, S Guyot, L Journaux, and P Molin, Microsc Microanal 23(1) (2017) 11-21

In this article, an automatic method for pseudo-circular object detection and counting, based on the Gradient Hough Transform, is described. We use this computational method for detection and quantitative characterization of yeast cells in white light micrographs. We enhance the original Gradient Hough Transform, which is based on an accumulation process, by adding two data structures: a local contributor list (LCL) and a used contributor matrix (UCM). These two data structures record the history of the accumulation process and allow for accurate peak detection and exploitation by use of a "deconstruction" algorithm introduced in this paper. These changes help
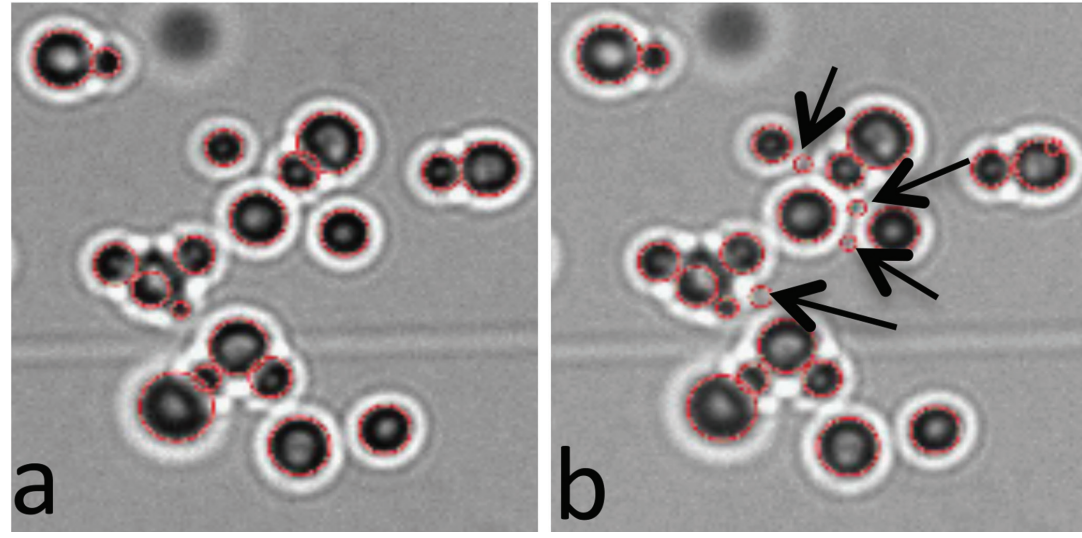

Yeast cell detection (a) using our method and (b) using classical circular Hough transform showing detection of spurious circles (arrows). make the algorithm a robust and precise method to automatically detect pseudo-circular objects in a micrograph. We present a cell-counting application using microbiology samples and, with a statistical study, prove that the automatic counts are relevant when compared to human counts and to the ground truth. Figure 1 presents comparison images illustrating yeast cell counting with our method and the classical circular Hough Transform method showing detection of spurious circles.

\section{A top journal in Microscopy}

\section{Published for the Microscopy Society of America}

Editor: John Mansfield, University of Michigan, USA

The only journal owned by scientists and published for scientists, Microscopy and Microanalysis provides original research papers in the fields of microscopy, imaging and compositional analysis. This distinguished international forum is intended for microscopists in both biology and materials science.

\section{Online submission at cambridge.org/mam/submit}

View the journal online at cambridge.org/mam

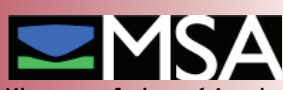

Microscopy Society of America

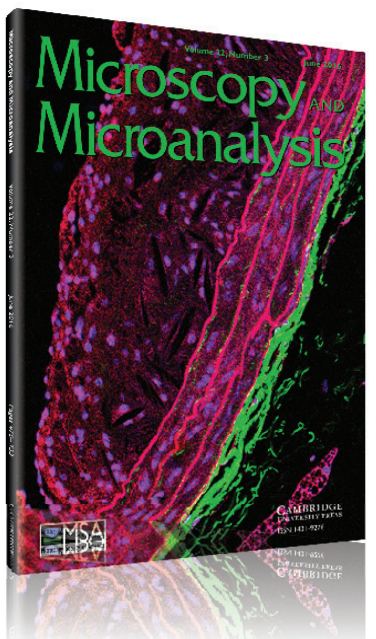

CAMBRIDGE UNIVERSITY PRESS 